(2) Open Access Full Text Article

\title{
Liraglutide Improves Non-Alcoholic Fatty Liver Disease In Diabetic Mice By Modulating Inflammatory Signaling Pathways
}

This article was published in the following Dove Press journal:

Drug Design, Development and Therapy

\author{
Ying Luo ${ }^{1, *}$ \\ Pijian Yang (D) ${ }^{1, *}$ \\ Zhengming $\mathrm{Li} \mathbb{D}^{2}$ \\ Yunchen Luo $\mathbb{D}^{2}$ \\ Jing Shen' \\ Ruwen $\mathrm{Li}^{1}$ \\ Hua Zheng ${ }^{3}$ \\ Yuzhen Liang ${ }^{2}$ \\ Ning Xia'
}

'Department of Endocrinology and Metabolism, The First Affiliated Hospital of Guangxi Medical University, Nanning 53002I, People's Republic of China; ${ }^{2}$ Department of Endocrinology and Metabolism, The Second Affiliated Hospital of Guangxi Medical University, Nanning 53002I, People's Republic of China; ${ }^{3}$ Life Sciences Institute, Guangxi Medical University, Nanning 53002I,

People's Republic of China

*These authors contributed equally to this work
Background: Many chronic metabolic diseases, such as obesity and type 2 diabetes (T2DM), are closely related to a chronic low-grade inflammatory state in tissues. The high prevalence of non-alcoholic fatty liver disease (NAFLD) in patients with T2DM is related to the role of inflammation in the disease. In this study, we investigated the role of liraglutide in improving lipid metabolism disorders and preventing their progression to NAFLD by modulating inflammatory signaling pathways, thereby providing new treatment options for NAFLD.

Methods: We designed a $2 \times 2$ factorial analysis experiment. A mouse model of NAFLD with T2DM was established by feeding the animals a high-fat diet (HFD). The NAFLD mice with HFD-induced diabetes were treated with liraglutide for 10 weeks. Hematoxylin and eosin staining, Oil Red $\mathrm{O}$ staining and electron microscopy were used to observe the accumulation of triglycerides in the liver. RT-PCR and Western blotting were used to analyze the expression of $\alpha$-SMA, IL- $1 \beta$, TNF- $\alpha, N F-\kappa B$ and the NF- $\kappa B$ inhibitory protein I $\kappa$ B in the liver at the gene and protein levels, respectively.

Results: Liraglutide reduced the body weight and fasting blood glucose levels of HFD-fed mice. The expression of $\alpha$-SMA, IL- $1 \beta$, TNF- $\alpha$, and NF- $\kappa B$ in the liver of HFD-fed mice was increased at the mRNA and protein levels, but liraglutide treatment decreased the expression of these molecules. The expression of IKB in the liver decreased at the mRNA and protein levels but was upregulated after liraglutide treatment.

Conclusion: Based on the current findings, liraglutide can significantly improve hepatic steatosis, primarily by downregulating the expression of inflammatory signaling mediators in the TNF- $\alpha$ pathway.

Keywords: liraglutide, nonalcoholic fatty liver disease, inflammatory signaling pathway

\section{Introduction}

NAFLD is a condition characterized by excess lipid deposition in the liver parenchyma and is associated with obesity, insulin resistance, and diabetes. In recent years, due to changes in lifestyle and dietary structure, the prevalence of NAFLD has increased rapidly along with metabolic diseases, such as obesity and type 2 diabetes (T2DM). The prevalence of NAFLD in adults is as high as $35 \%$ worldwide, ${ }^{1}$ while the prevalence is $29.62 \%$ in Asia. ${ }^{2}$ The incidence of obesity, hyperlipidemia, T2DM, and metabolic syndrome in patients with NAFLD is $51.3 \%, 69.2 \%, 22.5 \%$, and $42.5 \%$, respectively. ${ }^{3}$ Studies have shown that there is a correlation between NAFLD and T2DM. NAFLD increases the risk of T2DM, and T2DM also contributes to the progression of NAFLD. ${ }^{4}$ Hepatocytes exhibiting insulin resistance and
Correspondence: Yuzhen Liang; Ning Xia Email liangyuzhen26@163.com; xianinggxmu@163.com 
metabolic syndrome are key features of NAFLD. ${ }^{5}$ Rats with NAFLD showed high hepatic inflammation, necrosis and fatty infiltration. ${ }^{6}$ The disease is presumed to be mediated by the effects of the metabolic syndrome on the liver. The production of inflammation-inducing mechanisms and inflammatory cytokines production play a crucial role in the progression of NAFLD. ${ }^{7}$ Inflammation may affect the development of NAFLD due to its role in insulin resistance. Therefore, elucidating the detailed mechanism underlying the progression of NAFLD in patients with diabetes is important for disease treatment and drug development.

Glucagon-like peptide-1 (GLP-1) is a peptide hormone secreted by enteroendocrine $\mathrm{L}$ cells in response to nutrient intake. ${ }^{8}$ GLP-1 delays gastric emptying, stimulates insulin secretion and regulates the satiety signal in the central nervous system (CNS), all of which are beneficial for both obese individuals and patients with T2DM. In addition to blood glucose regulation, GLP-1 possesses antiapoptotic, anti-oxidant and anti-inflammatory properties ${ }^{9}$ that significantly reduce the production of reactive oxygen species in monocytes and reduce the mRNA expression of pro-inflammatory cytokines and various inflammatory mediators. ${ }^{10}$ GLP-1 also inhibits NF- $\mathrm{BB}$ activation and regulates the activity of natural killer cells in the pancreas, CNS and endothelial cells. ${ }^{9}$ Recently, GLP-1 receptors were reported to be expressed on human hepatocytes. ${ }^{11}$ In animal models of NAFLD or nonalcoholic steatohepatitis (NASH), glucose-induced GLP-1 secretion is significantly reduced, indicating a lack of GLP-1 signaling in patients with NAFLD. ${ }^{12}$ The number of GLP-1 receptors is reduced in liver biopsy specimens from patients with NASH compared to that in normal patients. ${ }^{13,14}$

Liraglutide is an analog of human glucagon-like peptide 1 with $97 \%$ structural homology to endogenous human GLP-1. Liraglutide is also one of the six GLP1-based drugs approved by the US Food and Drug Administration (FDA). Animal and human experiments have found that liraglutide has an effect on promoting weight loss and improving insulin resistance, liver lipid deposition and hepatic steatosis. ${ }^{15}$

However, the positive mechanical effect of liraglutide on hepatic steatosis remains unclear. Liraglutide stimulates glucose-dependent insulin secretion and modulates the levels of cardiovascular risk biomarkers, such as hs-CRP, IL-6 and TNF- $\alpha^{16}$ We hypothesized that liraglutide, as a GLP-1 ana$\log$, can inhibit the expression of inflammatory and proinflammatory cytokines, such as IL and TNF- $\alpha$ through the TNF- $\alpha$ inflammatory signaling pathway, thereby improving lipid metabolism and alleviating NAFLD development in diabetic mice.

In this study, we established a mouse model of NAFLD with T2DM by feeding the animals a high-fat diet (HFD). Using this mouse model, we investigated the molecular mechanism by which liraglutide regulates the inflammatory signaling pathway to prevent hepatic steatosis. Data from this study can provide useful information to improve our understanding of the pathophysiological effects of liraglutide on NAFLD by modulating inflammatory signaling pathways.

\section{Materials And Methods}

\section{Animal Model Preparation And Grouping}

A schematic of the experimental design is shown in Figure 1. Twenty-four 5-week-old male C57BL/6J mice were purchased from the Experimental Animal Center of Guangxi Medical University. All animals were housed in an SPF facility with a relative humidity of $30-70 \%$ and room temperature of $18-22{ }^{\circ} \mathrm{C}$. The mice were provided water food ad libitum. After 1 week of adaptive consumption of rodent feed ( $5 \%$ fat weight/food weight), the mice were randomly divided into a normal chow group (NC group) and a highfat diet group (HFD group) with 12 rats per group. The NC group was provided normal feed (5\% fat weight/food weight), while the HFD group was provided a high-fat diet (60\% fat weight/food weight, Ready Biotechnology Co., Ltd. Shenzhen, People's Republic of China). The body weight (BW) and fasting blood glucose (FBG) levels in samples collected from the tail vein were observed and recorded for all experimental mice at the same time every week. The hallmark of successful establishment of the NAFLD model is FBG levels $>13.9 \mathrm{mmol} / \mathrm{L}$ for three consecutive days and a BW that exceeds at least $20 \%$ of the normal body weight in the HFD-fed mice. ${ }^{17}$ At the beginning of the 10th week, the animals in the HFD group in which NAFLD was successfully established were randomly classified into 2 subgroups: a saline treatment group $(\mathrm{O}+\mathrm{S})$ and liraglutide treatment group $(\mathrm{O}+\mathrm{L})$. In addition, the $\mathrm{NC}$ control group was also randomly divided into 2 subgroups: a saline-treated group ( $\mathrm{N}$ $+\mathrm{S})$ and liraglutide-treated group $(\mathrm{N}+\mathrm{L})$. The liraglutidetreated groups were administered a daily subcutaneous injection of $0.2 \mathrm{mg} / \mathrm{kg}$ liraglutide, and the saline-treated groups were injected with a similar volume of physiological saline for a total of 10 weeks. At the end of the study, the mice were anesthetized and euthanized for blood and tissue collection. Part of the liver was frozen, and part of the liver was stored in 
Study design

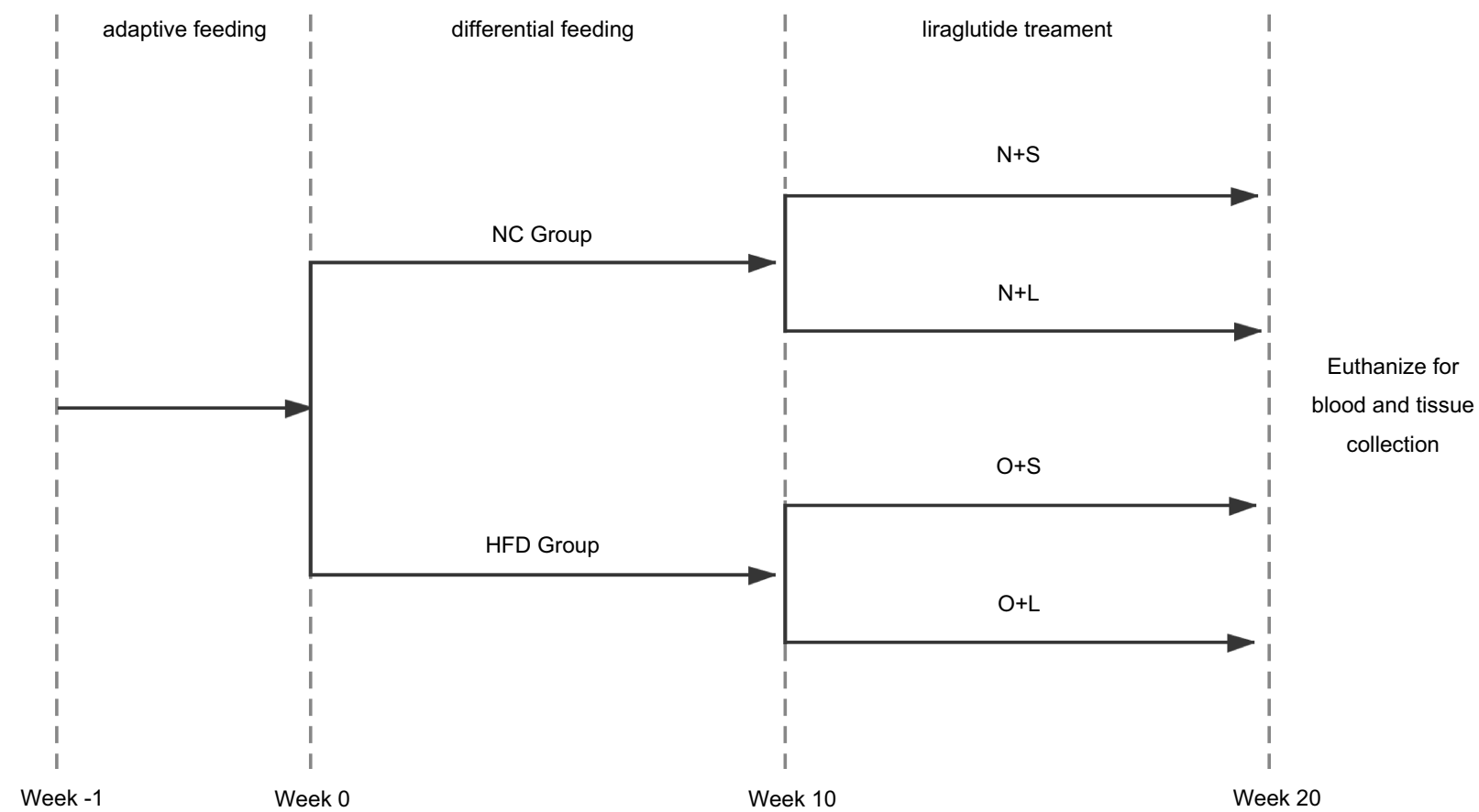

Figure I Study design. Steps used to analyze the effect of the liraglutide on NAFLD in the different groups.

a $4 \%$ buffered formalin solution. All experiments were approved by the Animal Ethics Committee of Guangxi Medical University. The conditions of the housing facilities, the care of laboratory animals and the animal experimental procedures were consistent with the national standard "Laboratory Animal-Guideline for Ethical Review of Animal Welfare" (GB/T35892-2018) of China.

\section{Biochemical Analysis}

The body weight and liver wet weight were determined. The blood glucose levels were measured using a blood glucose meter (Johnson \& Johnson, New Brunswick, NJ, USA).

\section{Histological Examination}

After the animals were euthanized, liver tissue was removed and fixed with a $4 \%$ neutral buffered formalin solution for 24 h. After paraffin embedding, tissue sections were cut into sections with a thickness of $5 \mu \mathrm{m}$, deparaffinized in xylene and rehydrated through a series of decreasing concentrations of ethanol. The sections were stained with hematoxylin and eosin (HE), which demarcates fat degeneration. Other samples were stained with Oil Red O, which stains lipid droplets and is used to visualize triglyceride accumulation in the liver. Images were captured with an optical microscope (Olympus, Tokyo,
Japan) and electron microscope (Hitachi H-7650 transmission electron microscope). Masson's trichrome staining was used to visualize fibrosis in the liver. Images were captured with a light microscope (Olympus, Tokyo, Japan).

\section{Analysis Of Gene Expression In Liver Tissues}

Total RNA was extracted from liver tissues using an RNA Extraction Kit (Takara Bio, Shiga, Japan). Total RNA was reverse transcribed using PrimeScript RT Master Mix (Perfect Real Time) (Takara Bio, Shiga, Japan). The primer sequences used for PCR are shown in Table 1 (Invitrogen, Shanghai, China). The $\beta$-actin mRNA served as the internal control. Real-time PCR was performed using SYBR Green Master Mix (Roche Diagnostics, Indianapolis, USA) on an Applied Biosystems StepOnePlus Real-Time PCR System (Thermo Fisher Scientific, Foster City, USA). Gene expression is reported as $2^{-\Delta \Delta \mathrm{Ct}}$.

\section{Western Blot Analysis Of Liver Tissues}

Western blotting was performed as previously described. ${ }^{18}$ Proteins were extracted from liver tissues using RIPA buffer containing PMSF, and protein concentrations were quantified with the bicinchoninic acid (BCA) assay method using 
Table I Primers For Polymerase Chain Reaction(B-Actin, IL- I $\beta$, TNF- $\alpha$, NF- $к b, ~ І к B-\alpha)$

\begin{tabular}{|l|l|l|}
\hline Gene name & Forward Primer & Reverse Primer \\
\hline$\beta$-actin & 5'- GGCACCACACCTTCTACAATGAGC-3' & 5'- GATAGCACAGCCTGGATAGCAACG-3' \\
IL-I $\beta$ & 5'- TCGCAGCAGCACATCAACAAGAG-3' & 5'- TGCTCATGTCCTCATCCTGGAAGG-3' \\
TNF- $\alpha$ & 5'- AGCTGGTGGTGCCATCAGAGG-3' & 5'- TGGTAGGAGACGGCGATGCG-3' \\
NF-kb & 5'- AGGCTCCTGTGCGTGTCTCC-3' & 5'- TCGTCTGTATCTGGCAGGTACTGG-3' \\
IkB- $\alpha$ & 5'- TCCACTCCATCCTGAAGGCTACC-3' & 5'- AGGTCCACTGCGAGGTGAAGG-3' \\
$\alpha-S M A$ & 5'- TGCTGGACTCTGGAGATGGTGTG-3' & 5'- CGGCAGTAGTCACGAAGGAATAGC-3' \\
\hline
\end{tabular}

a BCA protein assay kit (Thermo Scientific). Proteins were separated on $12 \%$ sodium dodecyl sulfate-polyacrylamide gels and transferred to polyvinylidene difluoride membranes. The membranes were incubated with Tris-buffered saline containing $5 \%$ non-fat milk at $37^{\circ} \mathrm{C}$ for $2 \mathrm{~h}$. The membranes were then incubated with the following primary antibodies at $37^{\circ} \mathrm{C}$ for $1 \mathrm{~h}$ : $\alpha$-SMA mAb (14395-1-AP; Proteintech, USA), IL-1 $\beta$ (D6D6T) rabbit mAb (mouse-specific) (\#31202; Cell Signaling Technology), TNF- $\alpha$ (D2D4) XP rabbit mAb (mouse-specific) (\#11948; Cell Signaling Technology), NFкB p65 (D14E12) XP rabbit mAb (\#8242; Cell Signaling Technology), $\beta$-actin (\#4970; Cell Signaling Technology), Histone-H3 Antibody125 Publications (17168-1-AP; Proteintech, USA), or anti-IKB alpha [E130] (ab32518; Abcam). Goat anti-rabbit IgG H\&L (HRP) (ab205718; Abcam) was used as the secondary antibody. After washing with PBS, the membranes were incubated with the secondary antibodies at $37^{\circ} \mathrm{C}$ for $1 \mathrm{~h}$. The membranes were developed with an enhanced chemiluminescence (ECL) system (ProteinSimple, Santa Clara, CA, USA).

\section{Statistical Analysis}

The data were analyzed using the SPSS 24.0 statistical package (Chicago, USA). Analysis of variance (ANOVA) with a $2 \times 2$ factorial design was used to analyze the data. The data are presented as the means \pm SD. For all tests, $\mathrm{p}<0.05$ was considered statistically significant.

\section{Results}

\section{Liraglutide Improves Metabolism And Reduces Intrahepatocellular Lipid Accumulation In The Mouse Model Of HFD-Induced NAFLD}

We performed $\mathrm{HE}$ and Masson staining to investigate pathological changes in the liver tissue and Oil Red $\mathrm{O}$ staining and transmission electron microscopy to observe lipid accumulation in hepatocytes. Compared with the NC group, the BW and FBG level were significantly increased in the HFD group after 10 weeks of consumption of the high-fat diet $(\mathrm{P}<0.05)$ (Figure $2 \mathrm{~A}$ and B). Liver histopathology and HE staining showed hepatocyte swelling, elevated steatosis, rarefaction of the hepatocyte cytoplasm and clumped strands of intermediate filaments in the HFD-fed mice (Figure 2C-F) compared to the normal liver structure of the control group. In addition, Oil Red O staining and transmission electron microscopy revealed lipid droplets in hepatocytes in the liver of HFDfed mice (Figure 5). The data indicate the successful establishment of the mouse model of NAFLD with T2DM.

After successful model establishment, mice with NAFLD and T2DM were treated with subcutaneous injections of liraglutide or saline daily for 10 weeks. After treatment with liraglutide, the BW and FBG levels were significantly reduced in the $\mathrm{O}+\mathrm{L}$ group (Figure $3 \mathrm{~A}$ and $\mathrm{B}$ ). A subsequent factor analysis showed significant differences in BW and FBG levels between NC and HFD-fed mice and between the liraglutidetreated and saline-treated groups (Figure $4 \mathrm{~A}$ and $\mathrm{B}$ ).

The comparison of the results of the morphological examination between the subgroups is shown in Figure 5. The $\mathrm{N}+\mathrm{S}$ and $\mathrm{N}+\mathrm{L}$ groups exhibited a normal liver structure. The normal structure of the hepatic lobules of the $\mathrm{O}$ $+\mathrm{S}$ group was characterized by a large amount of fatty degeneration. In contrast, a significant decrease in the severity of fat accumulation was observed in the liver tissue of mice in the $\mathrm{O}+\mathrm{L}$ group. Lipid droplets and fibrosis were undetectable in the livers of mice in the $\mathrm{N}$ $+\mathrm{S}$ and $\mathrm{N}+\mathrm{L}$ groups but were visible in the livers of mice in the $\mathrm{O}+\mathrm{S}$ group. In contrast, the liver of the mice in the $\mathrm{O}+\mathrm{L}$ group showed a significant reduction in lipid droplet accumulation and disappearance of fibrosis.

\section{Analysis Of Gene Expression In Liver Tissues}

The expression of $\alpha$-SMA, IL- $1 \beta$, TNF- $\alpha$, NF- $\kappa$ B and I $\kappa$ B was measured at week 20 (Figure 6). The factorial 

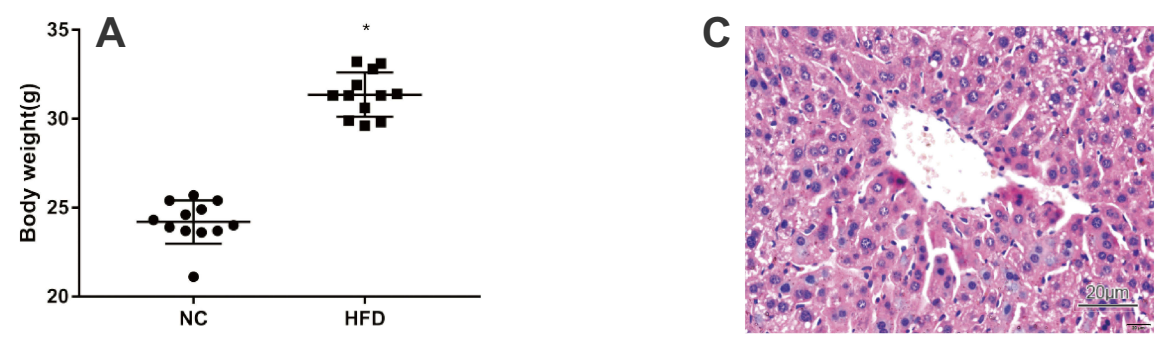

E
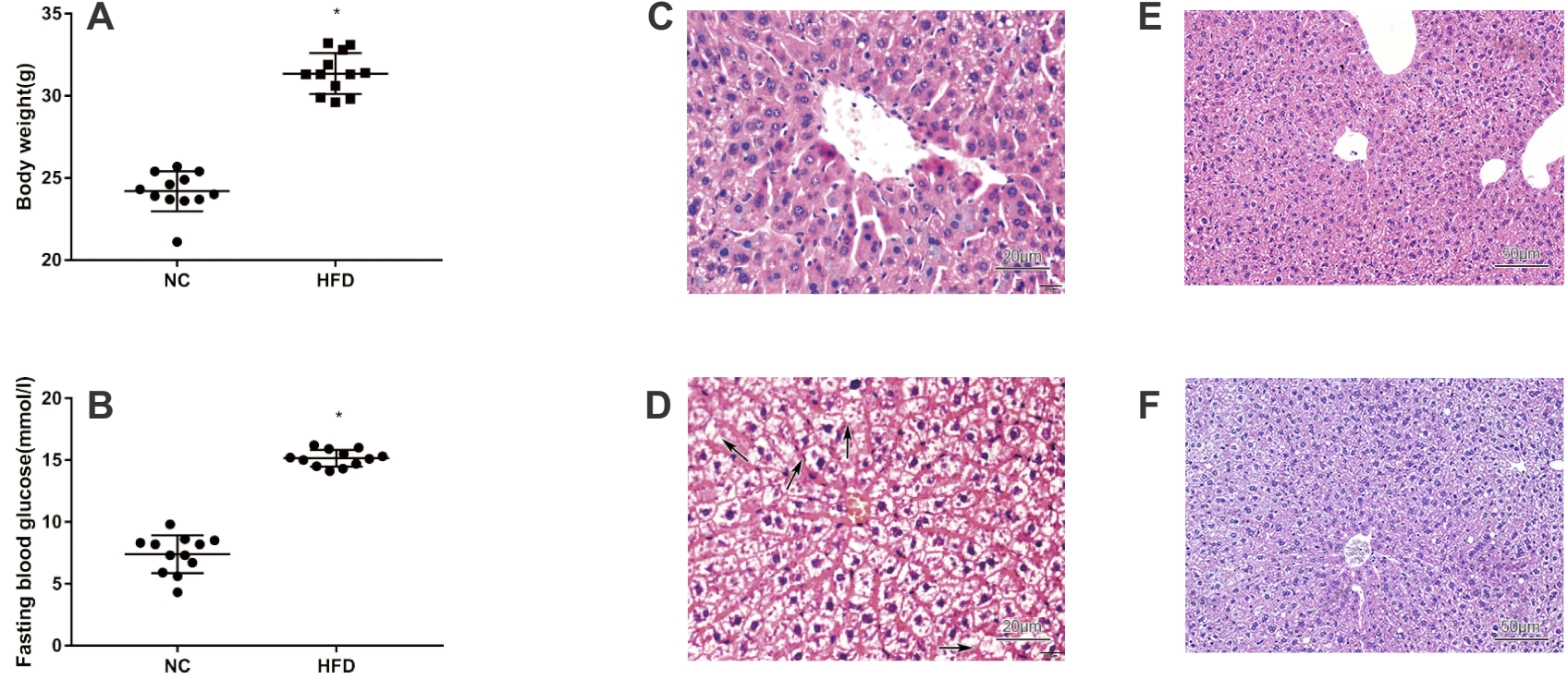

$\mathbf{F}$

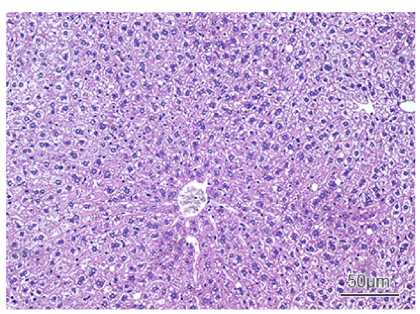

Figure 2 Physiological and histological assays of mice with HFD-induced NAFLD that were not treated with liraglutide. (A) Body weight of mice with HFD-induced NAFLD that were not treated with liraglutide (means $\pm S D, n=12$ ). (B) Fasting blood glucose levels in mice with HFD-induced NAFLD mice that were not treated with liraglutide (means \pm SD, $n=12$ ). Histological sections of liver tissues stained with HE from animals fed the normal rodent chow diet $(\mathbf{C}$ and $\mathbf{E})$ or HFD $(\mathbf{D}$ and $\mathbf{F})$ without the liraglutide treatment $(\mathrm{C}: \times 400, \mathrm{D}: \times 400, \mathrm{E}: \times 200, \mathrm{~F}: \times 200) . * \mathrm{P}<0.05$.
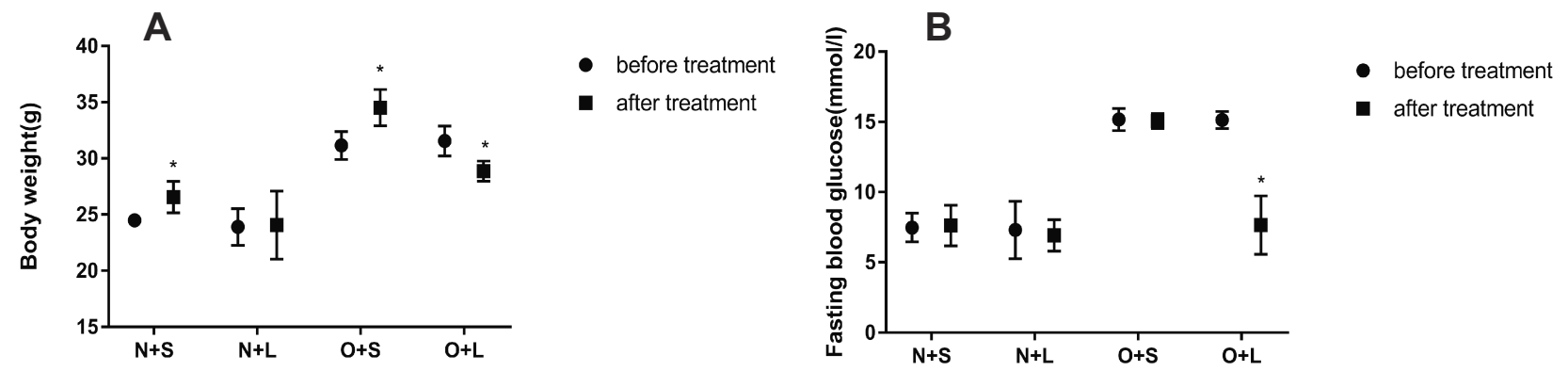

Figure 3 Effects of liraglutide on the body weight $(\mathbf{A})$ and blood glucose levels $(\mathbf{B})$ in the $N+S, N+L, O+S$ and $O+L$ groups $($ means $\pm S D, n=6)$. $* P<0.05$.

A

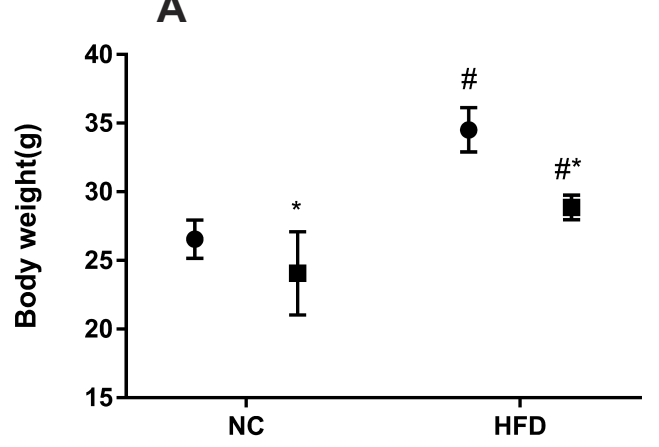

B

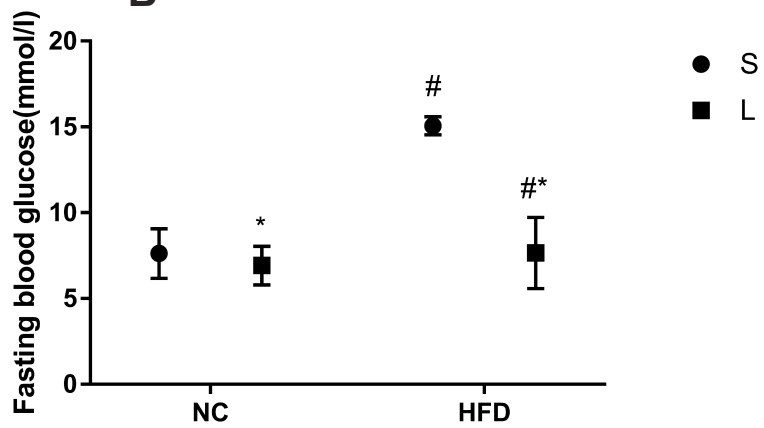

Figure 4 Effects of liraglutide on the body weight (A) and blood glucose levels (B) in mice fed the normal rodent chow diet and mice with HFD-induced NAFLD (means $\pm \mathrm{SD}, \mathrm{n}=6)$. ${ }^{*} \mathrm{P}<0.05$ for the comparison between saline and liraglutide treatments. ${ }^{\#} \mathrm{P}<0.05$ for the comparison between the NC and HFD groups.

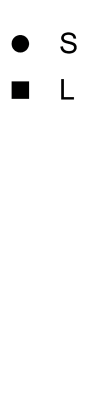




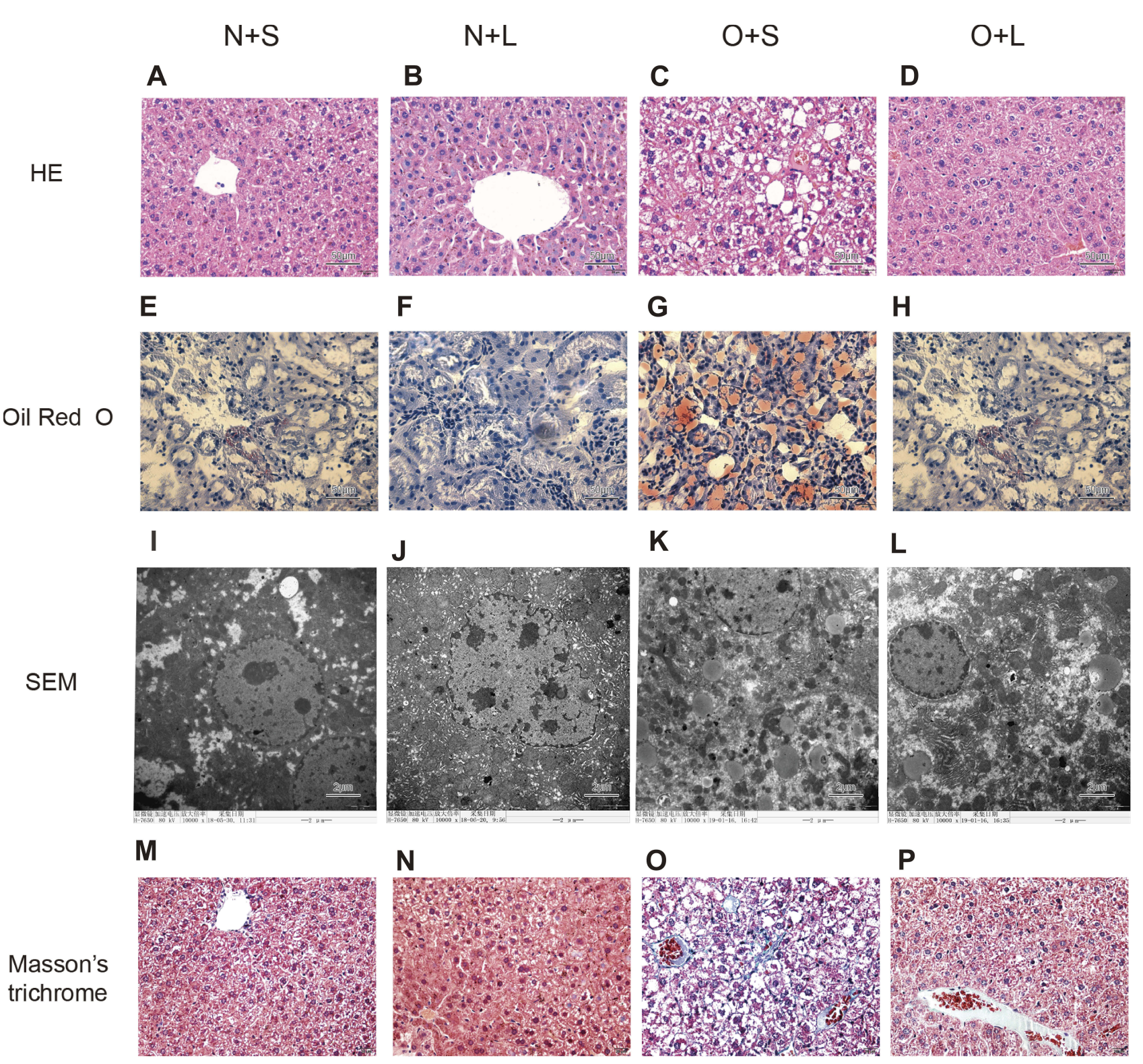

Figure 5 Histological analyses of liver tissues from each group stained with HE $(\times 400)$ and Oil Red $O(\times 400)$ and subsequent analysis using transmission electron microscopy $(\times 10,000)$. Images of HE staining (A-D), Oil Red O staining (E-H), SEM (I-L), and Masson's trichrome staining (M-P) in the N+S group (A, E, I and M), N+L group (B, F, J and $\mathbf{N})$, $\mathbf{O}+\mathbf{S}$ group $(\mathbf{C}, \mathbf{G}, \mathbf{K}$ and $\mathbf{O})$, and $\mathbf{O}+\mathbf{L}$ group $(\mathbf{D}, \mathbf{H}, \mathbf{L}$ and $\mathbf{P})$.

A

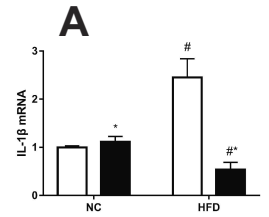

B

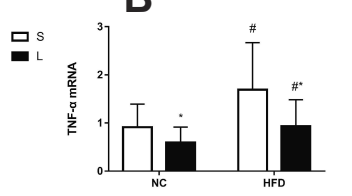

C

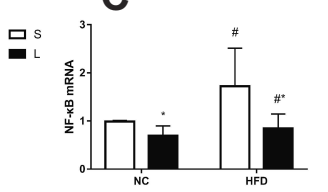

D

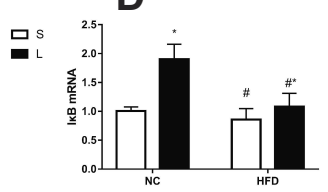

E

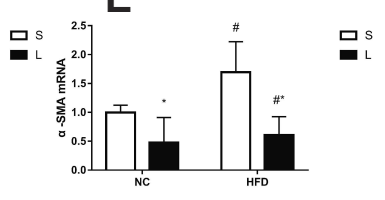

Figure 6 Expression of IL-I $\boldsymbol{\beta}(\mathbf{A})$, TNF- $\boldsymbol{\alpha}(\mathbf{B})$, NF-KB (C), IKB (D), and $\boldsymbol{\alpha}$-SMA (E) mRNAs in mice treated with liraglutide (L) or saline (S). Data are presented as the means $\pm S D$ ( $n=6$ mice per group). ${ }^{*} P<0.05$ for the comparison between saline and liraglutide treatments. ${ }^{\#} P<0.05$ for the comparison between the NC and HFD groups.

analyses revealed significant differences in the expression of $\alpha$-SMA, IL- $1 \beta$, TNF- $\alpha$, NF- $\kappa B$ and I $\kappa$ B mRNAs associated with the liraglutide treatment and saline treatment between the NC and HFD groups of mice. Compared to $\mathrm{NC}$ group, relative levels of the TNF- $\alpha, \mathrm{NF}-\mathrm{kB}$ and $\alpha-$ SMA mRNAs were significantly upregulated, and I $\mathrm{B}$ was 
downregulated in the HFD group $(\mathrm{P}<0.05)$. However, after treatment with liraglutide, levels of the TNF- $\alpha, \mathrm{NF}-\kappa \mathrm{B}$ and $\alpha$-SMA mRNAs were decreased $(\mathrm{P}<0.05)$, and levels of the IкB mRNA were increased $(\mathrm{P}<0.05)$. Compared to the $\mathrm{NC}$ group, the levels of the IL-1 $\beta$ mRNA were upregulated in the saline treatment groups but downregulated in the liraglutide treatment groups $(\mathrm{P}<0.05)$. When we compared different treatment groups, the levels of the IL-1 $\beta$ mRNA were increased in the $\mathrm{NC}$ groups and decreased in the HFD groups $(\mathrm{P}<0.05)$. In the NAFLD model, the levels of inflammatory factors were increased, but the liraglutide treatment effectively decreased the expression of the inflammatory factors.

\section{Western Blot Analysis Of Liver Tissues}

Proteins were extracted from liver tissues for Western blotting after 20 weeks (Figure 7). Compared to the NC group, the relative levels of the IL- $1 \beta$ protein were significantly increased, while the levels of the NF- $\kappa \mathrm{B}$ and $\mathrm{I} \kappa \mathrm{B}$ proteins were decreased, in the HFD group $(\mathrm{P}<0.05)$. The levels of the TNF- $\alpha$ protein were significantly increased by saline treatment and decreased by liraglutide treatment in the HFD groups compared to the NC groups. However, after treatment with liraglutide, the levels of the NF- $\mathrm{KB}$ and I $\kappa \mathrm{B}$ proteins were increased $(\mathrm{P}<0.05)$. The liraglutide treatment increased the levels of the IL- $1 \beta$, TNF- $\alpha$ and nuclear NF- $\kappa \mathrm{B}$ proteins in the NC groups and decreased their levels in the HFD groups $(\mathrm{P}<0.05)$. In mice with NAFLD, the NF- $\kappa B$ protein was transported to the nucleus from the cytoplasm, as cytoplasmic levels of the NF- $\kappa B$ protein were decreased and nuclear levels of the NF- $\kappa B$ protein were increased. These changes were effectively reversed by the liraglutide treatment. Compared with the NC group, the HFD group displayed increased $\alpha$-SMA levels $(\mathrm{P}<0.05)$. After treatment with liraglutide, the levels of the $\alpha$-SMA protein were decreased $(\mathrm{P}<0.05)$.

\section{Discussion}

NAFLD is the most prevalent chronic liver disease worldwide. NAFLD is primarily associated with obesity, diabetes, and metabolic syndrome, ${ }^{19,20}$ and no approved pharmacotherapy is available. Because the prevalence of NAFLD is increasing and is associated with poor outcomes, an effective drug must be developed to target the drivers and the associated complications, particularly in high-risk groups, such as older obese men and patients
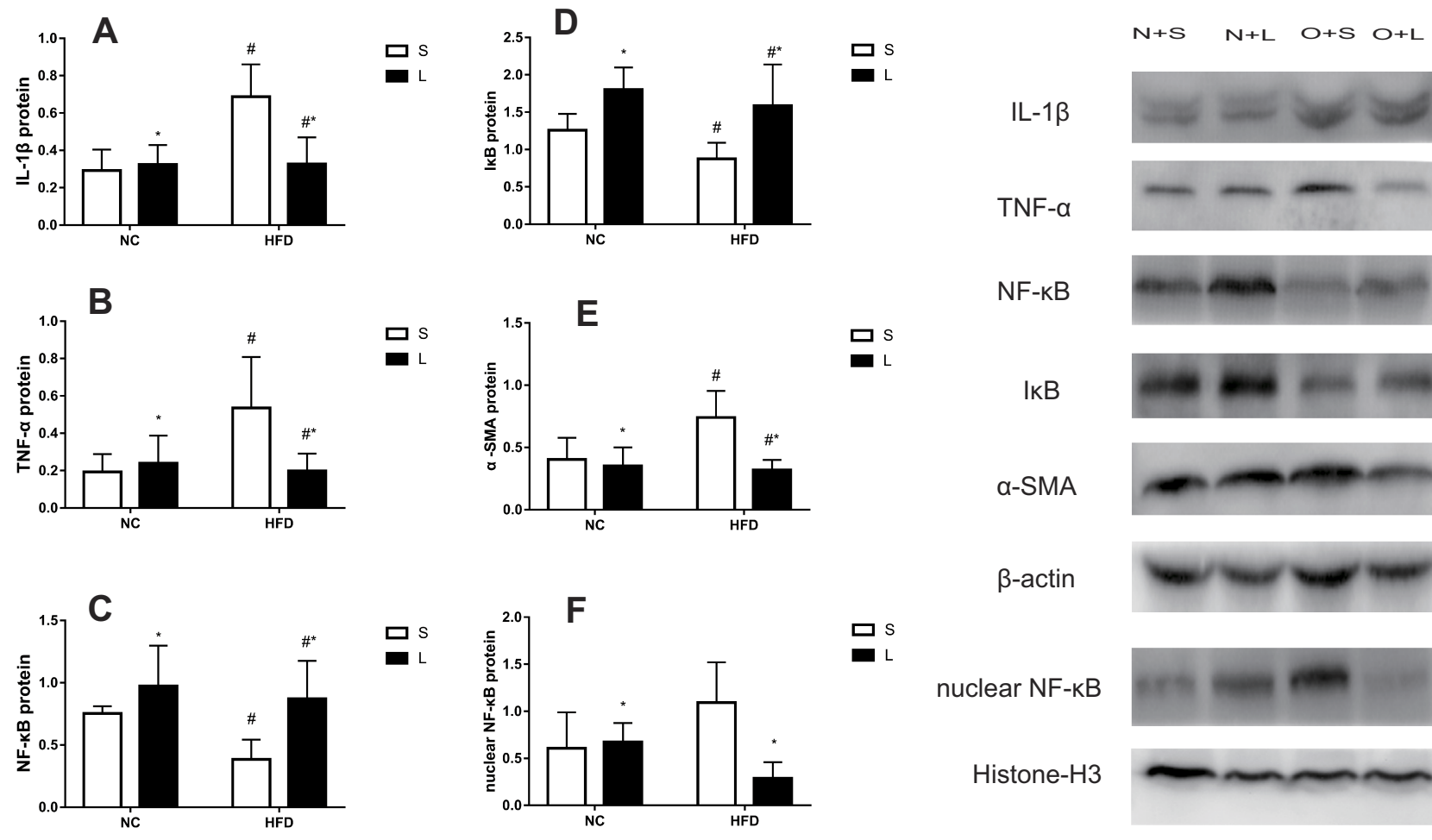

IKB

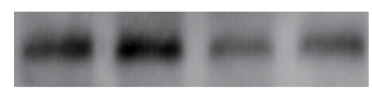

a-SMA

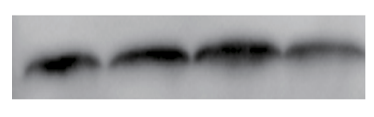

$\beta$-actin

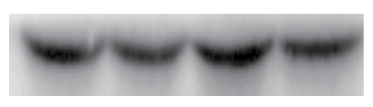

nuclear NF-kB

Histone-H3

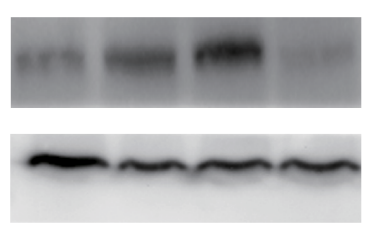

Figure 7 Levels of the IL-I $\beta(\mathbf{A})$, TNF- $\boldsymbol{\alpha}(\mathbf{B})$, NF- $\mathrm{KB}(\mathbf{C})$, IKB (D), $\boldsymbol{\alpha}-\mathrm{SMA}(\mathbf{E})$, and nuclear NF- $\mathrm{KB}(\mathbf{F})$ proteins in mice treated with liraglutide (L) or saline (S). Data are presented as the means $\pm S D$ ( $n=6$ mice per group). $* \mathrm{P}<0.05$ for the comparison between saline and liraglutide treatments. ${ }^{\#} \mathrm{P}<0.05$ for the comparison between the NC and HFD groups. 
with T2DM. Hepatocytes with insulin resistance and metabolic syndrome are key features of NAFLD. ${ }^{5}$ The disease is believed to be mediated by the effects of the metabolic syndrome on the liver. GLP-1RA, which has been approved for use in diabetes, has shown early efficacy in treating NASH and exerts beneficial cardiovascular or renal effects. ${ }^{21}$ Therefore, elucidating the detailed mechanism underlying the progression of NAFLD in patients with diabetes is important for disease treatment and drug development.

The peroxisome proliferator-activated receptors (PPARs) are members of the nuclear receptor superfamily that function as fatty acid-activated transcription factors. ${ }^{22}$ PPAR activation may limit inflammation and atherosclerosis. ${ }^{23}$ According to published data, PPAR- $\alpha$ and PPAR- $\gamma$ are related to lipid accumulation in the liver. Liraglutide reverses oxidative stress in diabetes by activating cardiac PPAR $\alpha$ to inhibit the expression of DGAT. ${ }^{24}$ In DIO rats, liraglutide reduces hepatic fatty acid flux by decreasing the expression of PPAR- $\gamma$ in the liver, which suggest a restoration of lipid homeostasis. ${ }^{25}$ These results help us to better understand the mechanisms regulating lipid metabolism that underlie the effectiveness of liraglutide as a treatment for diabetes and obesity. However, the relationship between the effects of liraglutide on regulating inflammation and lipid metabolism remains unclear.

Because a genetic model is more expensive and difficult to generate, the HFD-induced model is now more widely used to examine the pathophysiology of hepatic steatosis in an NAFLD model. ${ }^{26-28} \mathrm{~A}$ recent comparative pharmacological and histological study of biopsies from diet-induced and genetically obese mouse models confirmed the anti-NASH effects of liraglutide. ${ }^{29}$ In the present study, we built upon the foundation of our previous research, and the animal model of NAFLD induced by an HFD was successfully established after 10 weeks. ${ }^{30}$ After 10 weeks of treatment with clinically relevant concentrations of liraglutide, we observed therapeutic effects of liraglutide on NAFLD, including significant improvements in metabolic parameters, such as blood glucose and body weight. In addition, morphological observations, such as HE and Oil Red O staining, suggest that liraglutide effectively in relieving hepatic steatosis. Therefore, our results suggest that liraglutide exerts a beneficial effect on diabetes and obesity-related NAFLD. These results are consistent with previous studies. ${ }^{31,32}$

It is currently known that inflammation induction and inflammatory cytokines production play a crucial role in the progression of NAFLD. ${ }^{7}$ The IL-1 family of cytokines is one of the major drivers of inflammation in individuals with NAFLD. When these pro-inflammatory cytokines are activated, they disrupt insulin and lipid signaling pathways, affecting insulin sensitivity and lipid metabolism. ${ }^{33}$ IL-1 $\beta$ plays an important role in liver disease and is involved in all stages of the disease. IL- $1 \beta$ induces liver hepatic steatosis, inflammation and fibrosis by binding to and activating the IL-1 receptor, and IL-1 receptors are widely expressed in different hepatocyte subpopulations. ${ }^{34}$ IL-1 $\beta$ promotes hepatic steatosis by stimulating triglyceride and cholesterol accumulation in primary liver cells and inducing lipid droplet formation. ${ }^{7}$ IL-1 $\beta$ acts on hepatic sinusoidal endothelial cells and promotes hepatic inflammation by upregulating the expression of intercellular adhesion molecule 1 (ICAM-1), which causes neutrophils to accumulate in the liver. ${ }^{35}$ Taken together, IL- $1 \beta$ plays a major role in the development and progression of NAFLD from simple steatosis to steatohepatitis and liver fibrosis. ${ }^{36}$ In our study, the expression of IL- $1 \beta$ mRNA and protein showed a consistent trend. Compared with the untreated group, IL-1 $\beta$ expression was significantly increased in the NAFLD group, supporting the hypothesis that IL-1 $\beta$ is the main cause of liver inflammation in individuals with NAFLD. After treatment with liraglutide, IL-1 $\beta$ levels significantly decreased, suggesting that liraglutide can regulate the process of hepatic steatosis in subjects with NAFLD by regulating IL- $1 \beta$ expression.

The TNF family has very diverse functions in the body. The TNF family members all exhibit pro-inflammatory activity, in part through the activation of the transcription factor nuclear factor kappa B (NF-kB). TNF- $\alpha$ is a multifunctional cytokine that is mainly produced in adipocytes or peripheral tissues and is involved in apoptosis, cell differentiation and cell infiltration. Many inflammatory and autoimmune processes are also regulated by TNF- $\alpha$. TNF- $\alpha$ activates a transcriptional pathway that induces oxidative stress, which then interacts with inflammation to promote cell degeneration. ${ }^{37}$ TNF- $\alpha$ also causes insulin resistance and subsequently impairs glucose tolerance. ${ }^{38}$ In addition to insulin resistance, TNF- $\alpha$ may also affect glucose metabolism by affecting insulin secretion. ${ }^{39}$ Based on the results from our present study, TNF- $\alpha$ expression was increased in the NAFLD group and may be an important factor contributing to the inflammatory response of adipocytes in the NAFLD model. Liraglutide treatment can downregulate the expression of TNF- $\alpha$. 
NF- $\kappa B$ plays a key role in inflammation by inducing the transcription of pro-inflammatory genes. The activity of $\mathrm{NF}-\kappa \mathrm{B}$ is regulated by kappa $\mathrm{B}$ protein inhibitors (I $\mathrm{KB}$ )

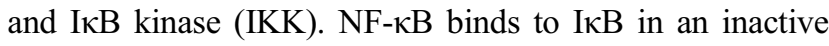
form in the cytoplasm. IKK-mediated phosphorylation of I $\kappa \mathrm{B}$ is an important step in NF- $\kappa \mathrm{B}$ activation because it causes the dissociation of NF- $\kappa B$ from I $\kappa \mathrm{B}$ and the translocation of NF- $\kappa B$ to the nucleus. ${ }^{40}$ As one of the most important regulators of proinflammatory gene expression, NF- $\mathrm{kB}$ regulates the synthesis of many inflammatory cytokines, including TNF- $\alpha$, IL- $1 \beta$, IL- 6 and IL- $8{ }^{40}$ According to another study, the main functions of GLP-1 are regulated by adenylate cyclase activation and cAMP production. ${ }^{41}$ In addition, the cAMP/PKA pathway regulates the inflammatory response of macrophages by inhibiting the production of pro-inflammatory cytokines. As shown in the study by Arakawa et al, the GLP-1 analog exenatide significantly reduces LPS-induced macrophage activation and TNF- $\alpha$ expression via the PKA/NF- $\kappa B$ signaling pathway. ${ }^{41}$ In addition, the activation of NF- $\mathrm{KB}$ has been reported to induce the transcription of pro-inflammatory genes, including TNF- $\alpha$, IL- $1 \beta$, IL- 6 and IL- $8^{40}$ Thus, GLP- 1 inhibits the inflammatory response of macrophages and the production of TNF- $\alpha$, IL- 6 and IL- $1 \beta$ via the cAMP/PKA/NF- $\kappa B$ signaling pathway. ${ }^{40,41}$ Liraglutide exerts marked antioxidative and anti-inflammatory effects on endothelial cells via inhibiting NF- $\mathrm{KB}$ activation and upregulating the expression of the IKB family. ${ }^{42}$ These results are consistent with our data. Liraglutide is an analog of GLP-1. In the present study, NF- $\mathrm{KB}$ mRNA was expressed at higher levels in the NAFLD group than in the normal control group. The NAFLD disease state may activate the NF- $\mathrm{KB}$ signaling pathway, stimulating the transcription of pro-inflammatory genes, including TNF- $\alpha$ and IL-1 $\beta$. However, the levels of these inflammatory markers can be effectively decreased by liraglutide treatment. The cytosolic levels of the NF- $\mathrm{kB}$ and I $\mathrm{B}$ proteins were decreased in the NAFLD group. The ubiquitination and proteasomal degradation of I $\kappa$ B directly activates dimeric NF- $\mathrm{KB}$, resulting in the translocation of $\mathrm{NF}-\mathrm{KB}$ to the nucleus and in the increase in the nuclear levels of this protein. These changes were reversed by liraglutide treatment. Based on our results, liraglutide can inhibit the NF- $\mathrm{KB}$ signaling pathway and decrease the production of various proinflammatory factors in a mouse model of NAFLD to reverse the resulting degeneration of hepatocytes. Therefore, liraglutide improves lipid metabolism in diabetic mice and prevents the development of NAFLD.

\section{Conclusions}

Liraglutide improves metabolism and significantly improves hepatic steatosis in the NAFLD model, primarily by downregulating the expression of inflammatory mediators in the TNF- $\alpha$ signaling pathway.

\section{Acknowledgment}

This work has been financially supported by the National Natural Science Foundation of China (Grant No.81760146).

\section{Disclosure}

The authors report no conflicts of interest in this work.

\section{References}

1. Vernon G, Baranova A, Younossi ZM. Systematic review: the epidemiology and natural history of non-alcoholic fatty liver disease and non-alcoholic steatohepatitis in adults. Alimentary Pharmacology \& Therapeutics. 2011;34(3):274-285.

2. Li J, Zou B, Yeo YH, et al. Prevalence, incidence, and outcome of non-alcoholic fatty liver disease in Asia, 1999-2019: a systematic review and meta-analysis. Lancet Gastroenterol Hepatol. 2019;4 (5):389-398. doi:10.1016/S2468-1253(19)30039-1

3. National Workshop on Fatty L, Alcoholic Liver Disease CSoHCMA, Fatty Liver Expert Committee CMDA. [Guidelines of prevention and treatment for nonalcoholic fatty liver disease: a 2018 update]. Zhonghua gan zang bing za zhi = Zhonghua ganzangbing zazhi = Chinese journal of hepatology. 2018;26(3):195-203.

4. Ballestri S, Zona S, Targher G, et al. Nonalcoholic fatty liver disease is associated with an almost twofold increased risk of incident type 2 diabetes and metabolic syndrome. Evidence from a systematic review and meta-analysis. Journal of Gastroenterology and Hepatology. 2016;31(5):936-944.

5. Younossi ZM. Non-alcoholic fatty liver disease _-2013; A global public health perspective. Journal of hepatology. 2019;70(3):531544.

6. Chen Z, Jain A, Liu H, Zhao Z, Cheng K. Targeted drug delivery to hepatic stellate cells for the treatment of liver fibrosis. $J$ Pharmacol Exp Ther. 2019;370(3):695-702. doi:10.1124/ jpet.118.256156

7. Negrin KA, Roth Flach RJ, DiStefano MT, et al. IL-1 Signaling in Obesity-Induced Hepatic Lipogenesis and Steatosis. PLOS ONE. 2014;9(9):e107265.

8. Holst JJ. The physiology of glucagon-like peptide 1. Physiol Rev. 2007;87(4):1409-1439. doi:10.1152/physrev.00034.2006

9. Campbell JE, Drucker DJ. Pharmacology, physiology, and mechanisms of incretin hormone action. Cell Metab. 2013;17(6):819-837. doi:10.1016/j.cmet.2013.04.008

10. Pugazhenthi U, Velmurugan K, Tran A, Mahaffey G, Pugazhenthi S. Anti-inflammatory action of exendin- 4 in human islets is enhanced by phosphodiesterase inhibitors: potential therapeutic benefits in diabetic patients. Diabetologia. 2010;53(11):2357-2368. doi:10.1007/ s00125-010-1849-y

11. He Q, Sha S, Sun L, Zhang J, Dong M. GLP-1 analogue improves hepatic lipid accumulation by inducing autophagy via AMPK/mTOR pathway. Biochemical and Biophysical Research Communications. 2016;476(4):196-203.

12. Bernsmeier C, Meyer-Gerspach AC, Blaser LS, et al. Glucoseinduced glucagon-like Peptide 1 secretion is deficient in patients with non-alcoholic fatty liver disease. PLoS One. 2014;9(1):e87488. doi:10.1371/journal.pone.0087488 
13. Gupta NA, Mells J, Dunham RM, et al. Glucagon-like peptide-1 receptor is present on human hepatocytes and has a direct role in decreasing hepatic steatosis in vitro by modulating elements of the insulin signaling pathway. Hepatology. 2010;51(5):1584-1592. doi:10.1002/hep.23569

14. Svegliati-Baroni G, Saccomanno S, Rychlicki C, et al. Glucagon-like peptide-1 receptor activation stimulates hepatic lipid oxidation and restores hepatic signalling alteration induced by a high-fat diet in nonalcoholic steatohepatitis. Liver Int. 2011;31(9):1285-1297. doi:10.1111/liv.2011.31.issue-9

15. Tong J, D'Alessio D. Give the receptor a brake: slowing gastric emptying by GLP-1. Diabetes. 2014;63(2):407-409. doi:10.2337/ db13-1764

16. Chen W-R, Shen X-Q, Zhang Y, et al. Effects of liraglutide on left ventricular function in patients with non-ST-segment elevation myocardial infarction. Endocrine. 2016;52(3):516-526.

17. Liang Y, Li Z, Liang S, et al. Hepatic adenylate cyclase 3 is upregulated by Liraglutide and subsequently plays a protective role in insulin resistance and obesity. Nutr Diabetes. 2016;6:e191.

18. Li Z, Yang P, Liang Y, et al. Effects of liraglutide on lipolysis and the AC3/PKA/HSL pathway. Diabetes, metabolic syndrome and obesity: Targets and Therapy. 2019;12:1697-1703.

19. Chalasani N, Younossi Z, Lavine JE, et al. The diagnosis and management of nonalcoholic fatty liver disease: practice guidance from the American Association for the Study of Liver Diseases. Hepatology. 2018;67(1):328-357. doi:10.1002/hep.29367

20. Mantovani A, Byrne CD, Bonora E, Targher G. Nonalcoholic fatty liver disease and risk of incident type 2 diabetes: a meta-analysis. diabetes care. 2018;41(2):372-382. doi:10.2337/dc17-1902

21. Sumida Y, Yoneda M. Current and future pharmacological therapies for NAFLD/NASH. $J$ Gastroenterol. 2018;53(3):362-376. doi:10.1007/s00535-017-1415-1

22. Willson TM, Brown PJ, Sternbach DD, Henke BR. The PPARs: from orphan receptors to drug discovery. J Med Chem. 2000;43 (4):527-550. doi:10.1021/jm990554g

23. Brown JD, Plutzky J. Peroxisome proliferator-activated receptors as transcriptional nodal points and therapeutic targets. Circulation. 2007;115(4):518-533. doi:10.1161/CIRCULATIONAHA.104.475673

24. Zhang Q, Xiao X, Zheng J, et al. Liraglutide protects cardiac function in diabetic rats through the PPARalpha pathway. Biosci Rep. 2018;38.

25. Decara J, Arrabal S, Beiroa D, et al. Antiobesity efficacy of GLP-1 receptor agonist liraglutide is associated with peripheral tissue-specific modulation of lipid metabolic regulators. Biofactors. 2016;42(6):600-611. doi:10.1002/biof.v42.6

26. Nagarajan P, Mahesh Kumar MJ, Venkatesan R, Majundar SS, Juyal RC. Genetically modified mouse models for the study of nonalcoholic fatty liver disease. World $J$ Gastroenterol. 2012;18 (11):1141-1153. doi:10.3748/wjg.v18.i11.1141

27. Arisqueta L, Navarro-Imaz H, Labiano I, Rueda Y, Fresnedo O. High-fat diet overfeeding promotes nondetrimental liver steatosis in female mice. Am J Physiol Gastrointest Liver Physiol. 2018;315(5): G772-G780. doi:10.1152/ajpgi.00022.2018
28. Xu H, Zhou Y, Liu Y, et al. Metformin improves hepatic IRS2/PI3K/ Akt signaling in insulin-resistant rats of $\mathrm{NASH}$ and cirrhosis. J Endocrinol. 2016;229(2):133-144. doi:10.1530/JOE-15-0409

29. Tolbol KS, Kristiansen MN, Hansen HH, et al. Metabolic and hepatic effects of liraglutide, obeticholic acid and elafibranor in diet-induced obese mouse models of biopsy-confirmed nonalcoholic steatohepatitis. World J Gastroenterol. 2018;24(2):179-194. doi:10.3748/wjg.v24.i2.179

30. Yang P, Liang Y, Luo Y, et al. Liraglutide ameliorates nonalcoholic fatty liver disease in diabetic mice via the IRS2/PI3K/Akt signaling pathway. Diabetes Metab Syndr Obes. 2019;12:1013-1021. doi:10.2147/DMSO.S206867

31. Ipsen DH, Rolin B, Rakipovski G, et al. Liraglutide decreases hepatic inflammation and injury in advanced lean non-alcoholic steatohepatitis. Basic Clin Pharmacol Toxicol. 2018;123(6):704-713. doi:10.1111/ bcpt.2018.123.issue-6

32. Zhu W, Feng PP, He K, Li SW, Gong JP. Liraglutide protects non-alcoholic fatty liver disease via inhibiting NLRP3 inflammasome activation in a mouse model induced by high-fat diet. Biochem Biophys Res Commun. 2018;505(2):523-529. doi:10.1016/j.bbrc.2018.09.134

33. Stienstra R, Joosten LAB, Koenen T, et al. The InflammasomeMediated Caspase-1 Activation Controls Adipocyte Differentiation and Insulin Sensitivity. Cell metabolism. 2010;12(6):593-605.

34. Tan Q, Hu J, Yu X, et al. The Role of IL-1 Family Members and Kupffer Cells in Liver Regeneration. BioMed Research International. 2016;2016:6.

35. Patrick AL, Rullo J, Beaudin S, Liaw P, Fox-Robichaud AE. Hepatic leukocyte recruitment in response to time-limited expression of TNF$\alpha$ and IL-1 $\beta$. American Journal of Physiology-Gastrointestinal and Liver Physiology. 2007;293(4):G663-G672.

36. Jain A, Barve A, Zhao Z, et al. Targeted delivery of an siRNA/PNA hybrid nanocomplex reverses carbon tetrachloride-induced liver fibrosis. Adv Ther. 2019;2(8):1900046. doi:10.1002/adtp.v2.8

37. Akash MSH, Rehman K, Liaqat A. Tumor Necrosis Factor-Alpha: Role in Development of Insulin Resistance and Pathogenesis of Type 2 Diabetes Mellitus. J Cell Biochem. 2018;119(1):105-110.

38. Hotamisligil GS, Shargill NS, Spiegelman BM. Adipose expression of tumor necrosis factor-alpha: direct role in obesity-linked insulin resistance. Science. 1993;259(5091):87-91.

39. Tsiotra PC, Tsigos C, Raptis SA. TNFalpha and leptin inhibit basal and glucose-stimulated insulin secretion and gene transcription in the HIT-T15 pancreatic cells. Int J Obes Relat Metab Disord. 2001;25 (7):1018-1026.

40. Tak PP, Firestein GS. NF-кB: a key role in inflammatory diseases. The Journal of Clinical Investigation. 2001;107(1):7-11.

41. Arakawa M, Mita T, Azuma K, et al. Inhibition of monocyte adhesion to endothelial cells and attenuation of atherosclerotic lesion by a glucagon-like peptide-1 receptor agonist, exendin-4. Diabetes. 2010;59(4):1030-1037. doi:10.2337/db09-1694

42. Shiraki A, Oyama J, Komoda H, et al. The glucagon-like peptide 1 analog liraglutide reduces TNF-alpha-induced oxidative stress and inflammation in endothelial cells. Atherosclerosis. 2012;221(2):375382 .

\section{Publish your work in this journal}

Drug Design, Development and Therapy is an international, peerreviewed open-access journal that spans the spectrum of drug design and development through to clinical applications. Clinical outcomes, patient safety, and programs for the development and effective, safe, and sustained use of medicines are a feature of the journal, which has also been accepted for indexing on PubMed Central. The manuscript management system is completely online and includes a very quick and fair peer-review system, which is all easy to use. Visit http://www. dovepress.com/testimonials.php to read real quotes from published authors. 